\title{
Improved Description of Charge-Transfer Potential Energy Surfaces via Spin-Component-Scaled CC2 and ADC(2) Methods
}

\author{
Attila Tajti,* Balázs Kozma, and Péter G. Szalay*
}

Cite This: J. Chem. Theory Comput. 2021, 17, 439-449

Read Online

ABSTRACT: The molecular level understanding of electronic transport properties depends on the reliable theoretical description of charge-transfer (CT)-type electronic states. In this paper, the performance of spin-component-scaled variants of the popular CC2 and $\mathrm{ADC}(2)$ methods is evaluated for CT states, following benchmark strategies of earlier studies that revealed a compromised accuracy of the unmodified models. In addition to statistics on the accuracy of vertical excitation energies at equilibrium and infinite separation of bimolecular complexes, potential energy surfaces of the ammonia-fluorine complex are also reported. The results show the capability of spin-component-scaled approaches to reduce the large errors of their regular counterparts to a significant extent, outperforming even the coupled-cluster single and double

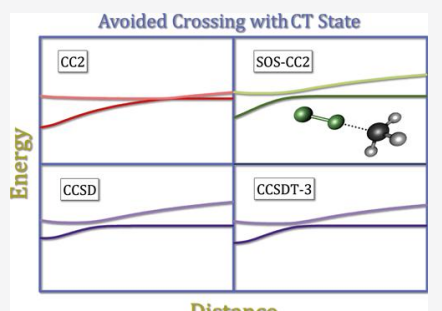

Distance method in many cases. The cost-effective scaled-opposite-spin variants are found to provide a remarkably good agreement with the CCSDT-3 reference data, thereby being recommended methods of choice in the study of charge-transfer states.

\section{INTRODUCTION}

The growing scientific interest in understanding molecular level electronic transport properties, molecular electronics, and related phenomena presents an important demand for a reliable theoretical description. The so-called charge-transfer (CT) states play a key role in such processes. These states are special types of electronically excited states formed via excitation of an electron from one part of the system to another, spatially distant area. Due to the presence of longrange correlation effects and, generally, the large size of the systems where these states can appear, describing CT states represents a big challenge for theoretical methods. Mean-field approaches, in particular time-dependent density functional theory (TDDFT) methods, are widespread choices in this area; ${ }^{1,2}$ however, their accuracy shows large variation with the functional and the system under inspection. ${ }^{3-5}$ Although many variants of TDDFT show good performance on CT states, $^{1,2,6-9}$ the reliability and consistency offered by correlated wave function methods are highly warranted in this field. The unfortunate scaling of the computational cost of wave function methods with the system size is a serious obstacle though, putting the focus on cheaper, approximate models. Popular techniques of this category are the various second-order approximate methods of the coupled-cluster (CC) family, mostly formulated as more or less systematic approximations to the $O\left(N^{6}\right)$ scaling coupled-cluster singles and doubles ${ }^{10}$ (CCSD) model at a lower computational cost. From the multitude of such theories, ${ }^{11}$ the CC2 $2^{12-14}$ and the closely related ${ }^{11,15}$ second-order algebraic diagrammatic construction $(\operatorname{ADC}(2))^{16-18}$ approaches are dominantly favored in applications thanks to their attractive, fifth-power computational scaling, efficient implementations and the latter being free from issues associated with the non-Hermitian nature of CC theory. The reliability and consistency of these models leave, as shown recently by $\mathrm{us}^{19-22}$ as well as by others, ${ }^{1,23-30}$ much to be desired: while vertical excitation energies of valence excitations are predicted with an outstanding accuracy, ${ }^{19,20}$ Rydberg states ${ }^{20,21}$ and potential energy surfaces $^{22}$ can be poorly described.

Moreover, we found recently ${ }^{31,32}$ that the regular CC2 and $\mathrm{ADC}(2)$ methods also handle the CT states with a very compromised accuracy. Our investigations, based on a set of local and CT states of bimolecular complexes, ${ }^{31}$ revealed a significant underestimation of the CT excitation energy at the equilibrium structure of these systems. In ref 32, it was also shown that many issues related to the inappropriate description of CT states are connected to the asymptotic behavior of the methods, i.e., the description of the charge transfer at infinite separation of the source and destination fragments. At these points, the charge transfer corresponds to a simultaneous ionization on the source and an electron attachment on the destination fragment, and the associated excitation energy of the CT state should precisely equal the sum of the ionization potential (IP) and electron affinity (EA) of the respective subsystems. This condition is, however, not fulfilled by methods that are not size extensive in the excited state, e.g., CCSD. ${ }^{33,34}$ (With CC methods, excited states are

Received: November 2, 2020

Published: December 16, 2020 
described either within the equation-of-motion $(\mathrm{EOM})^{35,36}$ or the linear response $e^{12,33,37,38}$ (LR) framework. Since these two provide identical excited state energies-the sole quantity discussed in the present work-we do not distinguish them and omit their notation for brevity.) In addition, the different descriptions of the electron correlation by the various methods influence the accuracy of the IP and EA values considerably, resulting in large errors of the asymptotic CT excitation energy for some models: both $\mathrm{CC} 2$ and $\mathrm{ADC}(2)$ were found to severely underestimate the reference values. ${ }^{32}$ These flaws seen on CT states are related to the case of Rydberg states in the sense that they both can likely be attributed to the inappropriate description of the electron moved far from its ground-state position. A significantly different accuracy of a method in the asymptotic and equilibrium regions has a consequence that the interconnecting potential energy surface (PES) of the CT state, normally characterized by the $1 / R$ attraction of the two ions, will also be inaccurate. Such surface scans revealed important problems with the reliability of the $\mathrm{CC} 2$ and $\mathrm{ADC}(2) \mathrm{CT}$ surfaces and the description of their avoided crossings with local excitations. ${ }^{32}$

The concept of spin-component scaling, originally proposed by Grimme and co-workers ${ }^{39,40}$ for the MP2 energy and later by Hättig and co-workers for $\mathrm{CC} 2$ and $\operatorname{ADC}(2),{ }^{41,42}$ introduces different multiplicative scaling factors $C_{\mathrm{SS}}$ and $C_{\mathrm{OS}}$ for the same-spin and opposite-spin integrals, respectively, in the energy expression and the terms of the effective Hamiltonian. The variants that are usually labeled as spincomponent-scaled (SCS) correspond to the parameters $C_{\mathrm{SS}}=$ $1 / 3$ and $C_{\mathrm{OS}}=6 / 5$, while the so-called SOS (scaled oppositespin) methods ${ }^{42}$ only retain the opposite-spin integrals with $C_{\mathrm{OS}}=1.3$. Although the determination of the above factors has been done rather empirically, Szabados ${ }^{43}$ elegantly showed that for the ground state, the SCS-MP2 scaling factors are practically the values that satisfy Feenberg's minimal condition in a two-parameter scaling of the zeroth-order Hamiltonian. The SOS methods are computationally very efficient as implementations with a computational scaling of no more than fourth power of the system size are possible. ${ }^{4-47}$ Nowadays, spin-component-scaled methods are available in many program packages ${ }^{48-51}$ and can be used for calculations on relatively large systems even on standard computational architectures.

The first experiences on the performance of spincomponent-scaled methods on excited states were rather mixed, ${ }^{45,52-54}$ which limited their popularity in applications. Recently, however, we pursued a quest to identify the terms responsible for the sometimes seriously bad performance of CC2 on Rydberg states ${ }^{21}$ and excited-state gradients, ${ }^{22}$ also evaluating spin-component-scaled methods in this context. ${ }^{55}$ It was found that errors of the regular CC2 model-as compared to either CCSD or a higher level reference-can be mitigated to a large extent by these approaches. ${ }^{55}$ Later, the analysis was extended to $\operatorname{ADC}(2)$ and its spin-component-scaled variants with a similar outcome, ${ }^{56}$ which did not come as surprise knowing the very close relationship of the CC2 and ADC(2) theories. 11,15

In the present work, we investigate the performance of spincomponent-scaled variants of CC2 and $\mathrm{ADC}(2)$ on CT states, encouraged by these promising results on Rydberg states and PESs. ${ }^{55,86}$ Since, in our earlier studies, no other pair of the $C_{S S}$ and $C_{O S}$ parameters was found to clearly outperform the common SCS and SOS methods, ${ }^{55}$ we only check these two approaches and refrain from the ambiguous, hardly justifiable tweaking of these parameters. In our examination, we follow the benchmarking procedures established in refs 31,32 by evaluating CT states of a set of two-component noncovalent molecular complexes in the energetically significant, low-lying area.

\section{METHODS}

We investigate the test systems from refs 31,32 , i.e., the ammonia-fluorine, acetone-fluorine, pyrazine-fluorine, ammonia-oxygendifluoride, acetone-nitromethane, ammoniapyrazine, pyrazine-pyrrole (in both stacked and H-bonded alignments), and ethylene-tetrafluoroethylene bimolecular complexes. On the various considerations about the selection of these systems and their excited states under inspection, the reader is referred to ref 31 . In that work, a benchmark set of 14 CT states was compiled from the low-lying states of the complexes via numerical definition of the CT characters (see below), while 41 other states were identified as local excitations. As spin-component-scaled approaches have already extensively been benchmarked on local excitations in refs 55 and 56, here, we only consider the CT states. The structures of the complexes were taken from that study, ${ }^{31}$ corresponding to the equilibrium distance between the two fragments.

As a reference, the high-level CCSDT- $3^{57}$ data from ref 31 were used, where the excellent accuracy of this method was also confirmed via comparisons to CCSDT results on selected systems.

In all calculations, we used the cc-pVDZ basis set of Dunning, ${ }^{58}$ which, despite its limited size and the lack of diffuse functions, was confirmed to provide reliable results for such comparisons. ${ }^{31}$ Core electrons were excluded from the correlation treatment, except for the calculation of $\omega$ descriptors and PESs (see below). All CC2, $\operatorname{ADC}(2)$, and spin-component-scaled calculations were performed with the TURBOMOLE $^{48,59}$ package, while CCSD and CCSDT-3 reference data were taken from ref 31.

To rely on a clear definition of the charge transfer nature of the states, as well as to free the analysis from ambiguities connected to the sometimes rather intricate mixing of dominant characters of different types in the wave functions (and their variation with the methods), the states are characterized using the $\omega$ descriptors of Plasser and coworkers. ${ }^{60-64}$ In this framework, the CT character $\omega_{\mathrm{CT}}$ (ranging from 0.0 of completely local states to 1.0 for pure $\mathrm{CT}$ ones) is defined as the weight of configurations in the wave function with charges separated on different fragments. The average exciton position $\omega_{\text {POS }}$ ranges from 1 to 2 in a twocomponent system, with the limits corresponding to local excitations on the first and the second fragments, respectively, while $\omega_{\mathrm{POS}} \approx 1.5$ to $\mathrm{CT}$ and completely delocalized (one-toone mixed) Frenkel resonance excitations. The participation ratio $\omega_{\mathrm{PR}}$ is close to 1 for both clear $\mathrm{CT}$ and local excitations, and in bimolecular complexes, $\omega_{\mathrm{PR}} \approx 2$ is typical in completely delocalized Frenkel-type and charge resonance states. The detailed introduction and demonstration of these quantities can be read in refs $31,32,60$, and 63 . The $\omega$ descriptors were evaluated with the TheoDORE program, ${ }^{63}$ using results from calculations performed with TURBOMOLE. We should note that unlike in the analysis of ref 31 where the full transition density matrix was used even at the CC2 level to calculate these numbers, this workflow approximates the respective transition matrix elements by the corresponding components 
of the one-particle solution vector of the excited state. Thus, in the present work, even CC2 descriptors can slightly differ from the ones obtained in the rigorous approach of ref 31 . The effects of this approximation are, however, minor (no more than $0.01,0.05$, and 0.04 in the CC2 $\omega_{\mathrm{POS}}, \omega_{\mathrm{PR}}$ and $\omega_{\mathrm{CT}}$ values, respectively) and thus do not influence the analysis significantly.

To check the problems associated with the asymptotic behavior, we calculate the CT excitation energies, as well as the associated ionization potential (IP) and electron affinity (EA) values with the spin-component-scaled methods, and compare them to the respective CCSDT-3 data and the results of the unscaled variants. The IP and EA calculations were done by the continuum orbital strategy described in ref 65, allowing for the evaluation of these quantities with any method able to predict excitation energies. This approach makes it possible to treat all ionized states on an equal footing in a closed-shell framework, potentially providing more accurate associated transition moments. ${ }^{66}$ It was successfully employed previously by us ${ }^{32,66}$ even at the TDDFT level and does not formally require a computer code to specifically support it: by including a separate, molecule-centered function in the basis set with a negligibly low exponent, ionized states are obtained in the excitation energy calculation as excitations to or from this orbital. The asymptotic excitation energies $\left(E_{\infty}^{\text {exc }}\right)$ were obtained at 10,000 bohrs of intermolecular separation, where the interaction energy of the two charges is already negligible (below $0.003 \mathrm{eV}$ ).

The effects on the CT potential energy surface are examined on a system where the various issues were presented and explained previously, ${ }^{32}$ the lowest totally symmetric CT state of the ammonia-fluorine complex and its interaction with a totally symmetric locally excited state.

\section{RESULTS AND DISCUSSION}

Identification of the CT States. To evaluate a performance of a theoretical method by comparison to results obtained with higher-level reference data, it is vital to ensure that the correct samples are compared in a statistical manner. While establishing a benchmark set for $\mathrm{CT}$ states in our previous work, ${ }^{31}$ it was revealed that forming a set of states that can be considered of CT type in all situations is not at all trivial and, due to the frequent mixing of CT and other (usually local valence) contributions in the wave function, requires one to follow a clear numerical definition in the classification of the states. To this end, threshold criteria for the $\omega$ descriptors (see above) prove to be an effective strategy. ${ }^{31}$ In that work, states with $\omega_{\mathrm{CT}}>0.5$ (with $\omega_{\mathrm{CT}}$ evaluated at the CCSD level) were considered as charge transfer, while all others were considered as local. (To also reflect a "mixed" nature of some states, the $\omega_{\mathrm{CT}}<0.1,0.1 \leq \omega_{\mathrm{CT}}<0.9$, and $0.9 \leq \omega_{\mathrm{CT}}$ categories were used for analysis as well.) Nevertheless, if different methods treat the various contributions in the wave function differently (as it is usually the case), the values of the $\omega$ descriptors can also be considerably different, potentially causing a state classified as CT by the CCSD measures to be a local excitation with that particular method. As including such states in a sideby-side comparison might provide misleading results, we first have to verify that an identical set of states is being used in the statistical analysis. Note that we do not aim to exclude "mixed" states in general but rather to avoid ones with a significantly different constitution. (For a detailed description of the classification problems that arise with CT states, the reader is referred to sections 4.1 and 4.2 of ref 31 ).

On Figure 1, the $\omega_{\mathrm{CT}}$ and $\omega_{\mathrm{PR}}$ characters are shown for all methods. The plots indicate a considerable variation of the

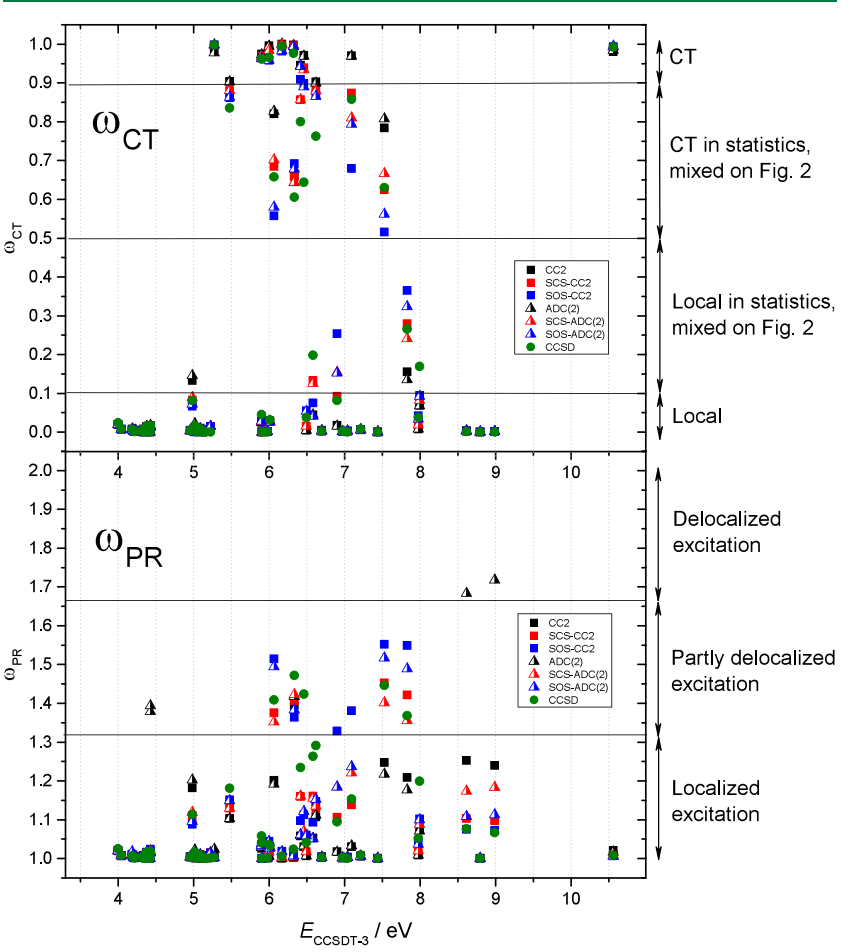

Figure 1. Charge-transfer character $\left(\omega_{\mathrm{CT}}\right.$, top panel) and the participation ratio $\left(\omega_{\mathrm{PR}}\right.$, lower panel) as functions of the CCSDT-3 vertical excitation energy $\left(E_{\text {CCSDT-3 }}\right)$ at equilibrium distance of the complexes, as obtained with different methods. See text for explanation.

character with the methods for many states, although the majority of states (especially the "clear" local and CT ones) remain of the same nature in all models. On Figure 1, a $\omega_{\mathrm{CT}}=$ 0.5 line is also shown, separating the CT and local states according to our definition. One can see that, fortunately, no states with the CCSD character (green dots) above this threshold fall below this line with any other method, so our definition provides the same set of CT states for all methods. However, for certain states, the spin-component-scaled variants (especially SOS-CC2 and SOS-ADC(2)) produce $\omega_{\mathrm{CT}}$ values very close to the decision threshold, while their unmodified counterparts show them to be much more of a clear CT or local type. This large shift in the wave function constitution caused by the spin-component scaling happens for states of "mixed" character, where the descriptors are very sensitive to the methods' ability to accurately provide the ratio of dominant contributions. A large difference between the CCSD and the unscaled CC2 and $\mathrm{ADC}(2)$ values is observed in these situations, and the spin-component-adjusted methods provide measures much closer to CCSD than their regular counterparts. This shows that they somewhat recover the "mixed" nature of these states, which are incorrectly predicted as "clean" by CC2 and $\mathrm{ADC}(2)$. Regardless of these phenomena, however, we can conclude that the benchmark set of $14 \mathrm{CT}$ states established in ref 31 can also be used to evaluate the SCS and SOS methods without modification. 
Table 1. Relative Error of the Calculated Excitation Energies $\left(\Delta_{\mathrm{CCSDT}-3}\left(E_{\mathrm{eq}}^{\mathrm{exc}}\right)\right.$, in eV) at Equilibrium Separation with Respect to CCSDT-3 Results for the Investigated CT States

\begin{tabular}{|c|c|c|c|c|c|c|c|c|}
\hline \multirow[b]{2}{*}{ system } & \multirow[b]{2}{*}{ state } & \multicolumn{7}{|c|}{$\Delta_{\text {CCSDT-3 }}\left(E_{\text {eq }}^{\mathrm{exc}}\right)$} \\
\hline & & $\mathrm{CC} 2$ & SCS-CC2 & SOS-CC2 & $\mathrm{ADC}(2)$ & SCS-ADC(2) & SOS-ADC $(2)$ & CCSD \\
\hline ammonia-fluorine & $2^{1} \mathrm{~A}_{1}$ & -0.65 & -0.30 & -0.12 & -0.63 & -0.27 & -0.10 & 0.28 \\
\hline acetone-fluorine & $3^{1} A^{\prime \prime}$ & -0.72 & 0.15 & 0.60 & -0.77 & 0.07 & 0.51 & 0.38 \\
\hline \multirow[t]{2}{*}{ pyrazine-fluorine } & $2^{1} \mathrm{~B}_{2}$ & -0.70 & 0.21 & 0.69 & -0.58 & 0.32 & 0.78 & 0.44 \\
\hline & $2^{1} \mathrm{~A}_{2}$ & -0.18 & 0.34 & 0.60 & -0.09 & 0.40 & 0.64 & 0.27 \\
\hline ammonia-oxygendifluoride & $4^{1} \mathrm{~A}^{\prime}$ & -0.76 & -0.03 & 0.43 & -0.80 & -0.11 & 0.33 & 0.24 \\
\hline acetone-nitromethane & $5^{1} \mathrm{~A}$ & -0.36 & 0.25 & 0.60 & -0.67 & -0.03 & 0.27 & 0.33 \\
\hline \multirow[t]{2}{*}{ ammonia-pyrazine } & $5^{1} \mathrm{~A}^{\prime}$ & -0.22 & 0.03 & 0.14 & -0.25 & 0.00 & 0.10 & 0.40 \\
\hline & $2^{1} \mathrm{~B}_{1}$ & -0.21 & 0.13 & 0.31 & -0.15 & 0.17 & 0.33 & 0.32 \\
\hline \multirow[t]{3}{*}{ pyrazine-pyrrole (H-bonded) } & $2{ }^{1} \mathrm{~A}_{1}$ & -0.25 & 0.08 & 0.25 & -0.24 & 0.08 & 0.24 & 0.33 \\
\hline & $3^{1} \mathrm{~A}_{1}$ & -0.24 & 0.10 & 0.27 & -0.22 & 0.10 & 0.26 & 0.30 \\
\hline & $2^{1} \mathrm{~A}^{\prime \prime}$ & -0.15 & 0.08 & 0.20 & -0.15 & 0.08 & 0.19 & 0.20 \\
\hline \multirow[t]{2}{*}{ pyrazine-pyrrole (stacked) } & $4^{1} \mathrm{~A}^{\prime}$ & -0.14 & 0.06 & 0.14 & -0.15 & 0.05 & 0.13 & 0.16 \\
\hline & $5^{1} \mathrm{~A}^{\prime}$ & -0.10 & 0.04 & 0.11 & -0.12 & 0.01 & 0.09 & 0.18 \\
\hline ethylene-tetrafluoroethylene & $5^{1} \mathrm{~B}_{1}$ & -0.40 & 0.16 & 0.45 & -0.28 & 0.27 & 0.56 & 0.31 \\
\hline mean & & -0.36 & 0.09 & 0.33 & -0.36 & 0.08 & 0.31 & 0.30 \\
\hline standard deviation & & 0.24 & 0.15 & 0.23 & 0.26 & 0.17 & 0.24 & 0.08 \\
\hline $\max$ & & -0.10 & 0.34 & 0.69 & -0.09 & 0.40 & 0.78 & 0.44 \\
\hline $\min$. & & -0.76 & -0.30 & -0.12 & -0.80 & -0.27 & -0.10 & 0.16 \\
\hline
\end{tabular}

Equilibrium Excitation Energies. The errors of vertical excitation energies relative to the reference CCSDT- 3 values for the CT states investigated in ref 31 are presented in Table 1 and Figure 2. The values were obtained at the equilibrium intermolecular distances discussed in detail in ref 31 . It is apparent that the SCS-type variants SCS-CC2 and SCS$\mathrm{ADC}(2)$ are equally very effective in reducing the underestimation of the CT excitation energies by their unscaled counterparts: the mean errors are no larger than $0.09 \mathrm{eV}$, and even the maximum errors $(0.34$ and $0.40 \mathrm{eV})$ are lower than the respective CCSD value. The SOS-type approaches, however, show a less appealing statistics: they turn the general underestimation of the equilibrium CT energies into a mean overestimation of similar magnitude which, although does not exceed considerably the CCSD result, comes with a much larger standard deviation with individual errors up to $+0.78 \mathrm{eV}$. Thus, as it is also apparent from the yellow (and orange) bars on the first and third row panels of Figure 2, the SOS methods do not seem to outperform the regular CC2 and $\mathrm{ADC}(2)$ methods in this regard. The effect we see here is most likely a systematic upshift of CT excitation energies by spincomponent scaling, similar to what was observed previously on Rydberg states. ${ }^{55,56}$ In this sense, the SOS set of parameters corresponds to a larger modification of the original formulae than the SCS one, thereby shifting the values further in the same direction. However, unlike to the case of Rydberg states where this results in about the same relatively low mean absolute error for both the SCS and SOS models, for CT states, only the SCS parametrization predicts the excitation energies with a good accuracy and the SOS one overestimates them considerably.

Asymptotic Limits. Statistics on the IPs of the source fragments, the EAs of the destination fragments, and the asymptotic CT excitation energies is given in Table 2, as a comparison to the respective CCSDT-3 results. (The full set of values is presented in the Supporting Information). As it was shown in ref 31 , CCSDT-3 provides these values with very little deviation from the full CCSDT ones, thereby being a reliable choice as a reference in the current investigation. Table
2 shows the significant underestimation of both the IP and EA values by the unscaled $\mathrm{CC} 2$ and $\mathrm{ADC}(2)$ models (with a striking similarity between these two), already discussed in ref 32 on a few selected states. As a consequence of this underestimation, the $\mathrm{CT}$ excitation energy at infinite separation is underestimated even more severely, almost by 1 $\mathrm{eV}$. The large associated standard deviations $(0.30$ and $0.34 \mathrm{eV}$ for CC2 and $\mathrm{ADC}(2)$, respectively, compared to the $0.06 \mathrm{eV}$ of CCSD) reflect a substantial inconsistency of this behavior.

The application of spin-component scaling to CC2$(\mathrm{ADC}(2))$ reduces the IP underestimations considerably, by $40(48) \%$ with the SCS and by $60(62) \%$ with the SOS parameters, respectively. The EA values are improved to an even larger extent, reducing the mean error by at least $76 \%$ (SCS-CC2), up to a nearly perfect agreement with CCSDT-3 in the case of SOS-ADC(2). Similar improvements are seen on the infinite separation CT energies, where the SOS variants show absolute mean errors of less than $0.1 \mathrm{eV}$, considerably outperforming even CCSD in this regard, although they still underestimate the reference value compared to the overestimation seen for CCSD. The standard deviations of all three quantities are also reduced considerably (by up to $59 \%$ in the case of SOS-CC2) with the scaled models but clearly do not approach the remarkable consistency of CCSD where no SD value exceeds $0.07 \mathrm{eV}$. Nevertheless, the spin-component scaling turns out quite effective in eliminating the severe errors of $\mathrm{CC} 2$ and $\mathrm{ADC}(2)$ at the infinite separation limit.

However, the perhaps most important property of the asymptotic behavior of a theoretical method is how accurately the CT excitation energy agrees with the sum of the IP and EA values of the source and destination fragments, respectively. Statistics on the difference between these quantities is presented in Table 3. As it was already shown in ref 32, CCSDT-3 reproduces the theoretical asymptotic energy with a very low $(<0.03 \mathrm{eV})$ error, while CCSD, due to the lack of size extensivity, shows a remarkable overestimation with the mean error being as large as $0.33 \mathrm{eV}$. Unscaled CC2 and ADC(2) also have a positive yet significantly lower mean error of 0.19 eV. Compared to them, the spin-component-scaled methods 


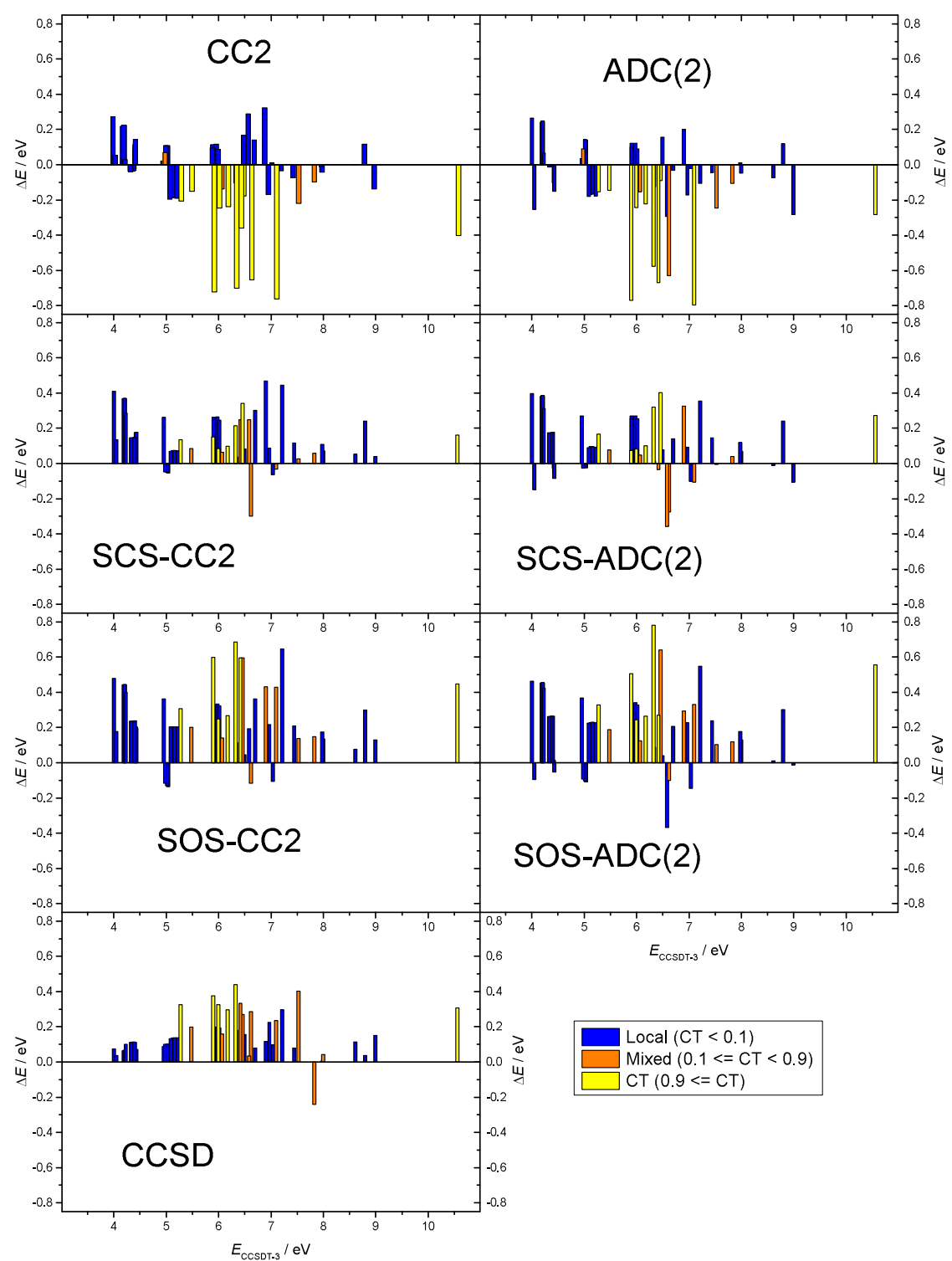

Figure 2. Error of the calculated excitation energy $(\Delta E)$ with respect to CCSDT-3 as a function of the CCSDT-3 excitation energy $\left(E_{\mathrm{CCSDT}-3}\right)$. The color of the bar shows the character of the respective excited state: blue for local states $\left(\omega_{\mathrm{CT}}<0.1\right)$, yellow for CT states $\left(\omega_{\mathrm{CT}} \geq 0.9\right)$, and orange for states of mixed character $\left(0.1 \leq \omega_{\mathrm{CT}}<0.9\right)$.

Table 2. Mean Errors (Relative to CCSDT-3 Reference Values, Standard Deviations in Parentheses) of the Ionization Potentials (IPs) of the Source Fragments, the Electron Affinities (EAs) of the Destination Fragments, and the Vertical Excitation Energies of the Investigated CT States at 10,000 bohr Separation $\left(E_{\infty}^{\text {exc }}\right)^{a}$

\begin{tabular}{llllllll} 
parameters & \multicolumn{1}{c}{ CC2 } & SCS-CC2 & SOS-CC2 & ADC $(2)$ & SCS-ADC(2) & SOS-ADC $(2)$ & CCSD \\
$\Delta_{\text {CCSDT-3 }}$ (IP) & $-0.58(0.31)$ & $-0.35(0.18)$ & $-0.23(0.13)$ & $-0.55(0.34)$ & $-0.33(0.22)$ & $-0.21(0.19)$ & $-0.01(0.07)$ \\
$\Delta_{\text {CCSDT-3 }}$ (EA) & $-0.49(0.18)$ & $-0.12(0.11)$ & $0.07(0.15)$ & $-0.53(0.17)$ & $-0.17(0.11)$ & $0.01(0.15)$ & $-0.04(0.03)$ \\
$\Delta_{\text {CCSDT-3 }}\left(E_{\infty}^{\text {exc }}\right)$ & $-0.90(0.30)$ & $-0.35(0.13)$ & $-0.07(0.15)$ & $-0.91(0.34)$ & $-0.38(0.19)$ & $-0.10(0.20)$ & $0.27(0.06)$
\end{tabular}

${ }^{a}$ All values are given in $\mathrm{eV}$ units.

Table 3. Statistics on the Deviation $\left(E_{\infty}^{\text {exc }}-(I P+E A)\right.$, in eV) of the Vertical Excitation Energies of the Investigated CT States at 10,000 bohr Separation from the Sum of the IP (Source Fragment) and EA (Destination Fragment) Values

$\begin{array}{cccccccccc}\text { parameters } & \text { CC2 } & \text { SCS-CC2 } & \text { SOS-CC2 } & \text { ADC(2) } & \text { SCS-ADC(2) } & \text { SOS-ADC(2) } & \text { CCSD } & \text { CCSDT-3 } \\ \text { mean } & 0.19 & 0.14 & 0.11 & 0.19 & 0.14 & 0.12 & 0.33 \\ \text { SD } & 0.07 & 0.05 & 0.04 & 0.08 & 0.06 & 0.05 & 0.07 \\ \text { max. } & 0.30 & 0.20 & 0.17 & 0.31 & 0.23 & 0.19 & 0.01 \\ \text { min. } & 0.10 & 0.06 & 0.04 & 0.10 & 0.06 & 0.04 & 0.04\end{array}$




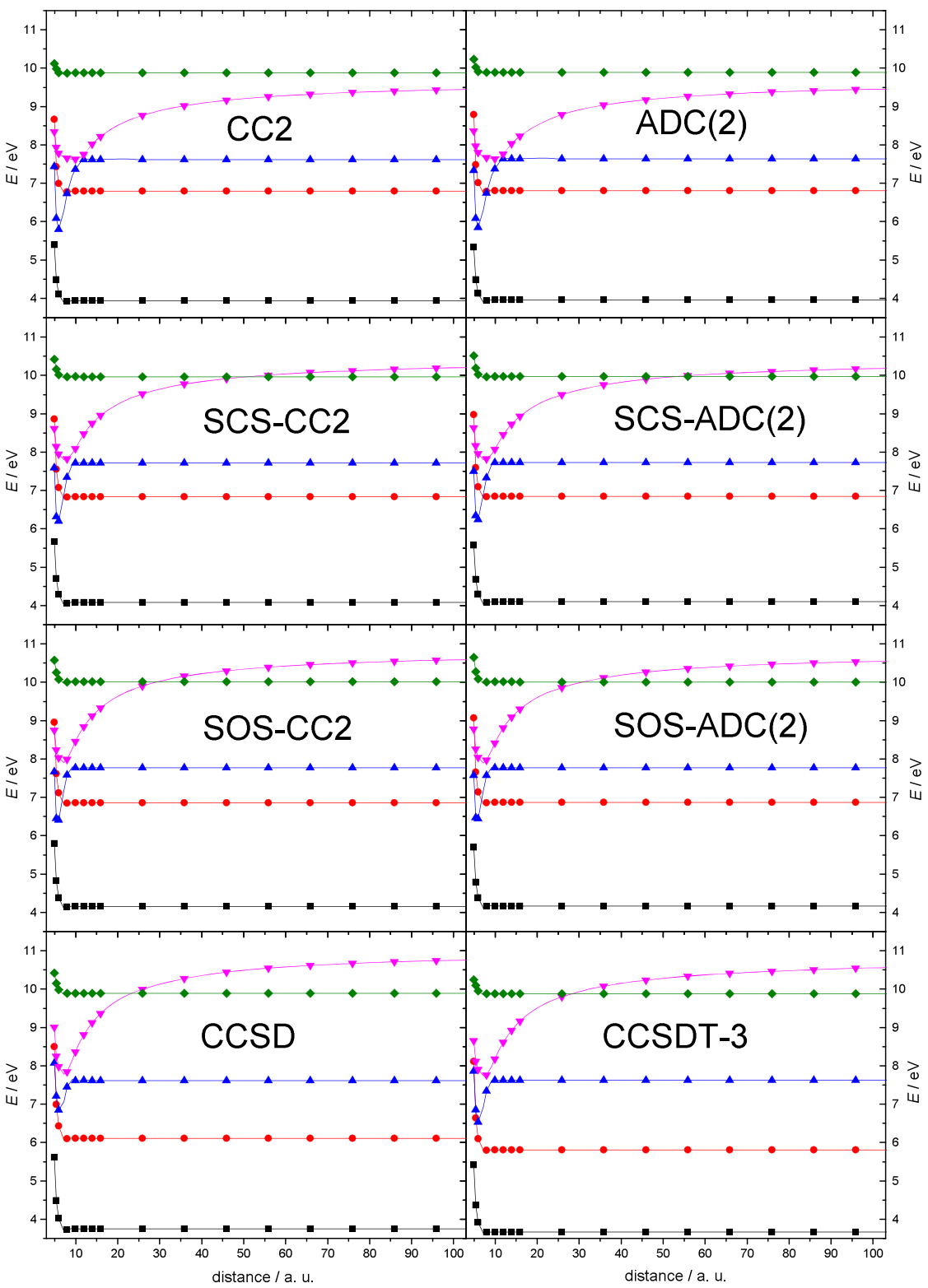

Figure 3. Potential energy of the five lowest $\left(1^{1} \mathrm{E}\right.$, black; $2^{1} \mathrm{E}$, red; $2^{1} \mathrm{~A}_{1}$, blue; $3^{1} \mathrm{~A}_{1}$, magenta; and $3^{1} \mathrm{E}$, green) excited states of the ammoniafluorine $\left(\mathrm{NH}_{3}-\mathrm{F}_{2}\right)$ complex as functions of the intermolecular separation.

behave noticeably better: the mean error is reduced to 0.14 and $0.11-0.12 \mathrm{eV}$ with the SCS and SOS variants, respectively. The fact that this is accompanied with low standard deviations (in fact, lower than those of the unscaled models) emphasizes the consistency of this improvement throughout the benchmark set.

Potential Energy Surfaces. While spin-component scaling seems thus to bring a spectacular improvement in the asymptotic behavior, at equilibrium intermolecular distances, the picture is more ambivalent. This can be an indication that investigating the potential energy surfaces and state characters along the separation of the components is, as seen already in ref 32, an important step in evaluating a method's performance in the description of CT states. To this end, we analyze these curves for the ammonia-fluorine bimolecular complex, one of the systems already studied in ref 32 . Figure 3 shows the potential energy curves of the low-lying excited states in the 0100 bohr range of the intermolecular separation. Beside the $2^{1} A_{1}$ and $3{ }^{1} A_{1}$ states (blue and magenta curves, respectively), which are a locally excited state on ammonia and a CT state, two local excitations on fluorine $\left(1^{1} \mathrm{E}\right.$ state, black curve and $2^{1} \mathrm{E}$ state, red curve) and one on ammonia $\left(3^{1} \mathrm{E}\right.$, green curve) are shown.

As the energy of the CT state has a very pronounced distance dependence ${ }^{32}$ generally characterized by the $1 / R$ decay of two interacting ions, one observes crossings between the CT and several other curves, but for symmetry reasons, interaction is only possible with the blue curve. As it was already discussed in ref 32 , the CCSD curves reproduce the features of higher-level ones very well, with the exception of the slightly overestimated asymptotic limit of the CT state. The unscaled CC2 and ADC(2), due to the sizeable underestimation of this limit, show a wrong order of states at large distances and the crossing of the $2{ }^{1} \mathrm{~A}_{1}$ and $3{ }^{1} \mathrm{~A}_{1}$ states happening at a much longer intermolecular distance. It is quite apparent how these erroneous properties of the CT curve are remedied if spin-component scaling is applied, while the locally excited states remain more or less unaffected. While the SCS- 


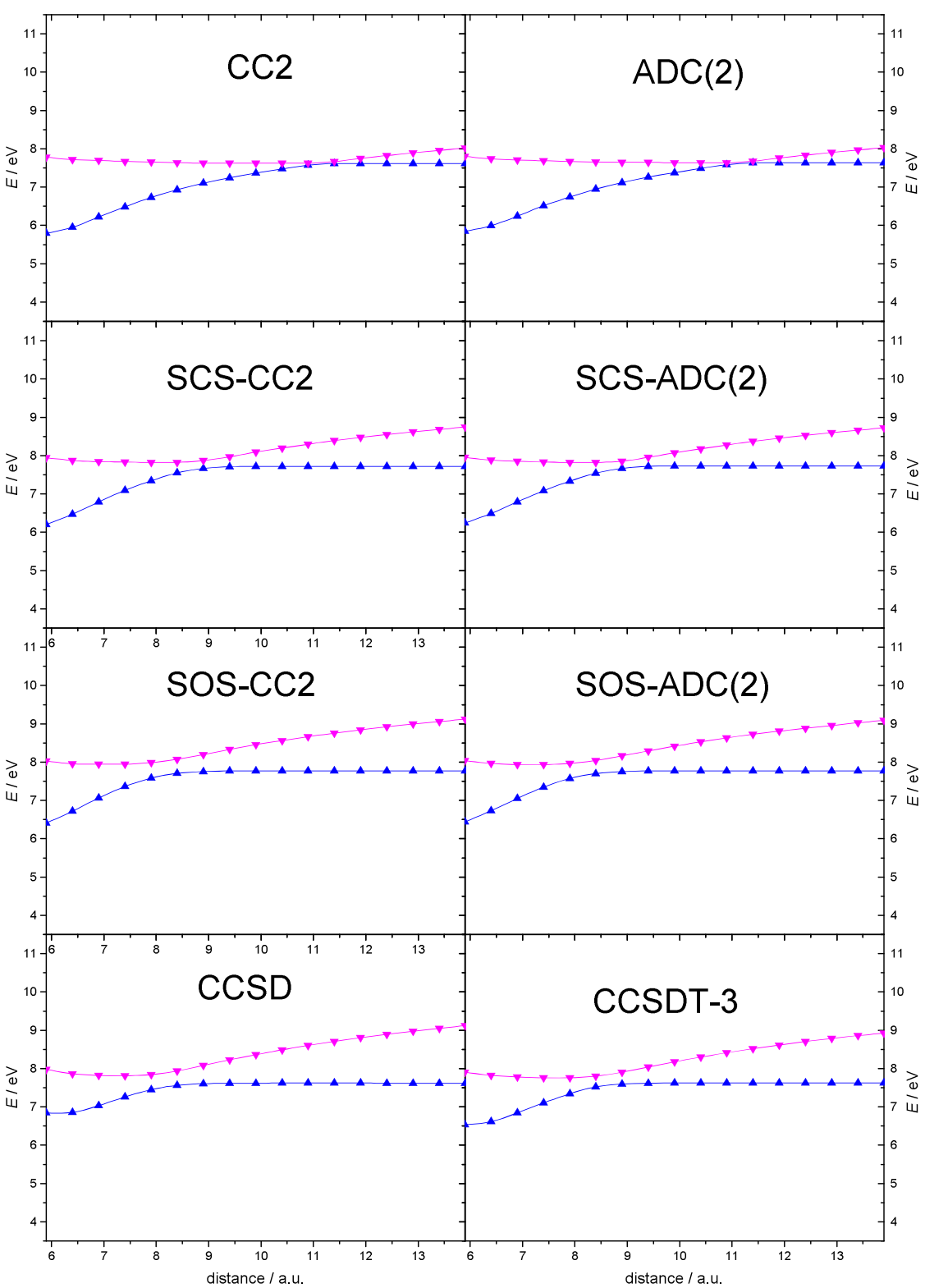

Figure 4. Crossing region of the energy curves for the $2^{1} \mathrm{~A}_{1}$ (blue) and $3{ }^{1} \mathrm{~A}_{1}$ (magenta) states of the ammonia-fluorine complex.

$\mathrm{CC} 2$ and SCS-ADC(2) results visually act like averages of those of CCSDT-3 and their unscaled counterparts, the SOS variants show curves remarkably similar to the reference method, providing, for example, a more accurate location of the crossing with the $3^{1} \mathrm{E}$ state than CCSD.

On Figures 4 and 5, the interaction regions of the CT state and the nearby totally symmetric local state are magnified. In this area, an avoided crossing between the two ${ }^{1} \mathrm{~A}_{1}$ states takes place, accompanied with the interchange of the state characters. The exact location of the avoided crossing and the shape of the surfaces in its vicinity are of special importance in applications that involve multiple interacting excited states, at least one of which having a significant CT character. In this regard, as it was shown first in ref 32, CCSD performs acceptably for this system, giving the location of the minimum separation of the states with a good accuracy; however, the gap of the avoided crossing is somewhat larger than what is provided by the triples methods. Both the regular $\mathrm{CC} 2$ and $\mathrm{ADC}(2)$ methods show a strikingly different picture here: the interaction of the states is essentially missing, resulting in a rapid swap of the CT character (see Figure 5) and a quasi-degenerate point, which is located at a more than $40 \%$ larger intermolecular distance than with CCSDT-3 or CCSD. In ref 32, it was shown that the missing interaction in the CC2 (and, due to the close relationship of the two theories, $\mathrm{ADC}(2))$ description is not caused by the larger distance per se but rather by the underestimated charge separation in these models. The introduction of spin-component scaling, again, turns out to be effective for correcting this qualitatively wrong behavior. SCS-CC2 and SCS-ADC(2) show a gap between the states, although much smaller than the reference method does, the minimum separation point also being at about $0.5 \mathrm{bohr}$ larger distance. Nevertheless, the $\omega_{\mathrm{CT}}$ character curves on Figure 5 (the respective plots of the $\omega_{\mathrm{PR}}$ and $\omega_{\mathrm{POS}}$ quantities can be found in the Supporting Information) reflect a correctly described interaction of the states, with the decays and elevations of the curves around the swapping point shaped similarly to CCSD. With the SOS models, an even larger 


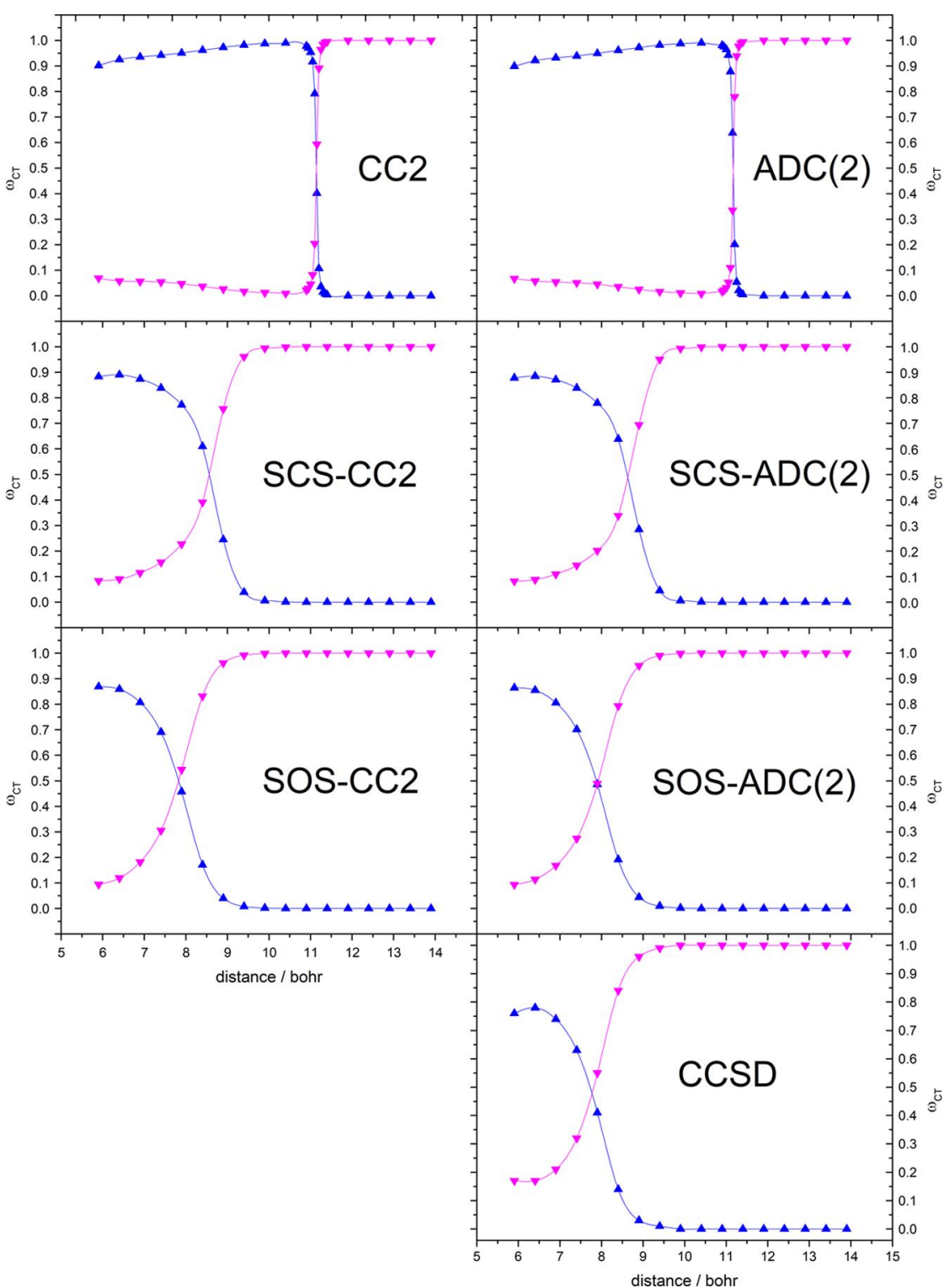

Figure 5. $\omega_{\mathrm{CT}}$ character (see Methods section) of the $2^{1} \mathrm{~A}_{1}$ (blue) and $3^{1} \mathrm{~A}_{1}$ (magenta) states of the ammonia-fluorine complex.

improvement is seen: both the size of the minimum energy gap and its position are very well reproduced; in fact, both SOS$\mathrm{CC} 2$ and SOS-ADC(2) outperform even CCSD in this regard.

It is thus hard to argue against the clear superiority of all spin-component-scaled models over their regular counterparts in the description of the CT surface, especially at the vicinity of the avoided crossing. Although we only discussed one CT state of a particular system here, due to the fact that the subpar performance of the unscaled methods stems from the improper description of the charge separation, ${ }^{32}$ it is reasonable to assume the generality of this finding.

\section{CONCLUSIONS}

In this work, we analyzed how spin-component scaling approaches impact the normally quite inaccurate description of charge transfer states by the CC2 and $\mathrm{ADC}(2)$ models. To this end, we followed the same benchmarking strategy established in earlier works, ${ }^{31,32}$ where this poor performance was shown the first time with calculations performed on a set of bimolecular complexes comparing the results to high-level $a b$ initio reference data. In this investigation, we once again confirmed the very close similarity of the CC2 and ADC(2) theories and the performance of their identically parametrized variants, a finding first established and explained in ref 56. Here, specifically looking at the CT states, we discovered practically no difference between any $\mathrm{CC} 2$ variant and its $\mathrm{ADC}(2)$ counterpart, thus favoring one model over the other would not be founded by our results.

From the spin-component scaling parameters, we checked the SCS and SOS variants and both turned out to reduce the large errors of the regular (unscaled) methods to a considerable extent, although their effectiveness depends somewhat on the situation under inspection. At the equilibrium separation of the complexes, the vertical excitation energies of CT states, underestimated severely by the standard approximate models, are very accurately predicted by SCSCC2 and SCS-ADC(2). The SOS methods turn this into a noticeable overestimation, which is, however, very close to the unapproximated CCSD result. The investigation of the asymptotic behavior, i.e., the results at the practically infinite 
separation of the fragments where the methods' performance in describing the ionization potentials and electron affinities is critical, revealed that spin-component-scaled techniques outperform not only the regular ones but even CCSD. This is most likely due to the better description of charge separation and a lower weight of size-inextensive terms in the wave functions. In this limit, the SOS variants show some degree of superiority compared to the SCS ones.

The region that interpolates between the equilibrium and asymptotic separations, i.e., the potential energy surface of the CT state and its interaction with other states, was inspected on a previously studied benchmark system, the ammonia-fluorine complex. Although one would normally warn against drawing general conclusions about a method's reliability by only looking at a particular example, the findings of this analysis are quite general as they stem from the inconsistent performance of $\mathrm{CC} 2$ and $\mathrm{ADC}(2)$ at large and short separations. In this test, as compared to CCSD or a higher level reference, both these methods turn out so bad that one should advise against their black-box application in studies where the right shape of CT potential energy surfaces and their interaction with other states can be of importance. ${ }^{32}$ Spin-component scaling, on the other hand, remedies the flaws very effectively: both the SCS and SOS parameters bring a noticeable improvement, the latter approaching the quality of the reference CCSDT-3 surfaces.

Our results, although showing a conclusive superiority of the spin-component-scaled approaches compared to their parent methods in the handling of CT states, do not generally favor either the SCS or SOS variants over the other. The $\omega$ characters obtained with the SCS parameters are, probably due to the more modest reshaping of the wave function, somewhat closer to the CCSD ones. In this sense, they act as more accurate in catching the correct character of the states. In the statistics of the vertical excitation energies, however, CCSD is better approached by the SOS variants. Whether the ability of approximate methods to reproduce the very systematic, albeit imprecise results of CCSD, or their absolute accuracysometimes benefiting from error cancellation effects-is considered more important, it is, to some extent, a matter of personal preference. Considering both the SCS and SOS parameter sets as empirical modifications, we do not see either as the one that should be clearly preferred for accuracy or consistency. The results where modified CC2 and ADC(2) methods act as superior to CCSD should also be regarded with awareness to the generally less systematic behavior of these models.

Nevertheless, from the perspective of applicability to large systems, the effectiveness of the SOS-CC2 and SOS-ADC(2) methods should not be ignored. These methods, as it was shown in refs 55, 56, not only offer a more consistent description of Rydberg states compared to regular CC2 and $\mathrm{ADC}(2)$ but also provide that of CT-type ones, while possessing an attractive $O\left(N^{4}\right)$ computational scaling with the system size. The results of this work should be an encouragement for considering spin-component-scaled approximate methods, in particular SOS-type ones on large systems, in excited state $a b$ initio applications in place of the regular variants or lower level mean-field models.

\section{ASSOCIATED CONTENT}

\section{Supporting Information}

The Supporting Information is available free of charge at https://pubs.acs.org/doi/10.1021/acs.jctc.0c01146.

Values of the $\omega_{\mathrm{CT}}, \omega_{\mathrm{POS}}$, and $\omega_{\mathrm{PR}}$ descriptors for all considered states; list of vertical excitation energies for $\mathrm{CT}$ and local states at equilibrium and infinite separations; individual ionization potentials and electron affinities, and plots of the state characters around the studied avoided crossing (PDF)

\section{AUTHOR INFORMATION}

\section{Corresponding Authors}

Attila Tajti - Institute of Chemistry, Laboratory of Theoretical Chemistry, ELTE Eötvös Loránd University, H-1518

Budapest, Hungary; 이이. orcid.org/0000-0002-7974-6141; Email: tat@chem.elte.hu

Péter G. Szalay - Institute of Chemistry, Laboratory of Theoretical Chemistry, ELTE Eötvös Loránd University, $H$ 1518 Budapest, Hungary; (1) orcid.org/0000-0003-18853557; Email: szalay@chem.elte.hu

\section{Author}

Balázs Kozma - Institute of Chemistry, Laboratory of Theoretical Chemistry, ELTE Eötvös Loránd University, H1518 Budapest, Hungary; (ㅇ orcid.org/0000-0002-12630371

Complete contact information is available at:

https://pubs.acs.org/10.1021/acs.jctc.0c01146

\section{Notes}

The authors declare no competing financial interest.

\section{ACKNOWLEDGMENTS}

This work has been funded by the National Research, Innovation and Development Fund (Nemzeti Kutatási Fejlesztési és Innovációs Hivatal-NKFIA) grant no. 124018.

\section{REFERENCES}

(1) Kümmel, S. Charge-Transfer Excitations: A Challenge for TimeDependent Density Functional Theory That Has Been Met. Adv. Energy Mater. 2017, 7, 1700440.

(2) Maitra, N. T. Charge transfer in time-dependent density functional theory. J. Phys.: Condens. Matter 2017, 29, 423001.

(3) Dreuw, A.; Weisman, J. L.; Head-Gordon, M. Long-range charge-transfer excited states in time-dependent density functional theory require non-local exchange. J. Chem. Phys. 2003, 119, 29432946.

(4) Dreuw, A.; Head-Gordon, M. Single-Reference ab Initio Methods for the Calculation of Excited States of Large Molecules. Chem. Rev. 2005, 105, 4009-4037.

(5) Zhao, Y.; Truhlar, D. G. Density Functional for Spectroscopy: No Long-Range Self-Interaction Error, Good Performance for Rydberg and Charge-Transfer States, and Better Performance on Average than B3LYP for Ground States. J. Phys. Chem. A 2006, 110, 13126-13130.

(6) Stein, T.; Kronik, L.; Baer, R. Reliable Prediction of Charge Transfer Excitations in Molecular Complexes Using Time-Dependent Density Functional Theory. J. Am. Chem. Soc. 2009, 131, 2818-2820.

(7) Kuritz, N.; Stein, T.; Baer, R.; Kronik, L. Charge-Transfer-Like $\pi$ $-\pi *$ Excitations in Time-Dependent Density Functional Theory: A Conundrum and Its Solution. J. Chem. Theory Comput. 2011, 7, $2408-2415$ 
(8) Ottochian, A.; Morgillo, C.; Ciofini, I.; Frisch, M. J.; Scalmani, G.; Adamo, C. Double hybrids and time-dependent density functional theory: An implementation and benchmark on charge transfer excited states. J. Comput. Chem. 2020, 41, 1242-1251.

(9) Hait, D.; Zhu, T.; McMahon, D. P.; Van Voorhis, T. Prediction of Excited-State Energies and Singlet-Triplet Gaps of ChargeTransfer States Using a Restricted OpenShell Kohn-Sham Approach. J. Chem. Theory Comput. 2016, 12, 3353-3359.

(10) Purvis, G. D., III; Bartlett, R. J. A Full Coupled-Cluster Singles and Doubles Model - the Inclusion of Disconnected Triples. J. Chem. Phys. 1982, 76, 1910-1918.

(11) Izsák, R. Single-reference coupled cluster methods for computing excitation energies in large molecules: The efficiency and accuracy of approximations. Wiley Interdiscip. Rev.: Comput. Mol. Sci. 2020, 10, No. e1445.

(12) Christiansen, O.; Koch, H.; Jørgensen, P. The Second-order Approximate Coupled-Cluster Singles and Doubles Model CC2. Chem. Phys. Lett. 1995, 243, 409-418.

(13) Hättig, C.; Weigend, F. CC2 excitation energy calculations on large molecules using the resolution of the identity approximation. $J$. Chem. Phys. 2000, 113, 5154-5161.

(14) Köhn, A.; Hättig, C. Analytic gradients for excited states in the coupled-cluster model $\mathrm{CC} 2$ employing the resolution-of-the-identity approximation. J. Chem. Phys. 2003, 119, 5021.

(15) Hättig, C. In Response Theory and Molecular Properties (A Tribute to Jan Linderberg and Poul Jorgensen); Jensen, H., Ed.; Advances in Quantum Chemistry; Academic Press, 2005; Vol. 50; pp. 37-60, DOI: 10.1016/S0065-3276(05)50003-0.

(16) Schirmer, J. Beyond the Random-Phase Approximation - A New Approximation Scheme for the Polarization Propagator. Phys. Rev. A 1982, 26, 2395-2416.

(17) Schirmer, J.; Trofimov, A. B. Intermediate state representation approach to physical properties of electronically excited molecules. J. Chem. Phys. 2004, 120, 11449-11464.

(18) Dreuw, A.; Wormit, M. The algebraic diagrammatic construction scheme for the polarization propagator for the calculation of excited states. WIREs Comput. Mol. Sci. 2015, 5, 82-95.

(19) Kánnár, D.; Szalay, P. G. Benchmarking Coupled Cluster Methods on Valence Singlet Excited States. J. Chem. Theory Comput. 2014, 10, 3757-3765.

(20) Kánnár, D.; Tajti, A.; Szalay, P. G. Accuracy of Coupled Cluster excitation energies in diffuse basis sets. J. Chem. Theory Comput. 2016, 13, 202-209.

(21) Tajti, A.; Szalay, P. G. Investigation of the Impact of Different Terms in the Second Order Hamiltonian on Excitation Energies of Valence and Rydberg States. J. Chem. Theory Comput. 2016, 12, 5477-5482.

(22) Tajti, A.; Stanton, J. F.; Matthews, D. A.; Szalay, P. G. Accuracy of Coupled Cluster Excited State Potential Energy Surfaces. J. Chem. Theory Comput. 2018, 14, 5859-5869.

(23) Sauer, S. P. A.; Schreiber, M.; Silva-Junior, M. R.; Thiel, W. Benchmarks for Electronically Excited States: A Comparison of Noniterative and Iterative Triples Corrections in Linear Response Coupled Cluster Methods: CCSDR(3) versus CC3. J. Chem. Theory Comput. 2009, 5, 555-564.

(24) Silva-Junior, M. R.; Sauer, S. P. A.; Schreiber, M.; Thiel, W. Basis set effects on coupled cluster benchmarks of electronically excited states: CC3, CCSDR (3) and CC2. Mol. Phys. 2010, 108, 453-465.

(25) Sous, J.; Goel, P.; Nooijen, M. Similarity transformed equation of motion coupled cluster theory revisited: a benchmark study of valence excited states. Mol. Phys. 2014, 112, 616-638.

(26) Harbach, P. H. P.; Wormit, M.; Dreuw, A. The third-order algebraic diagrammatic construction method $(\mathrm{ADC}(3))$ for the polarization propagator for closed-shell molecules: Efficient implementation and benchmarking. J. Chem. Phys. 2014, 141, No. 064113.

(27) Jacquemin, D.; Duchemin, I.; Blase, X. 0-0 Energies Using Hybrid Schemes: Benchmarks of TD-DFT, CIS(D), $\operatorname{ADC}(2), \mathrm{CC} 2$, and BSE/ GWformalisms for 80 Real-Life Compounds. J. Chem. Theory Comput. 2015, 11, 5340-5359.

(28) Piecuch, P.; Hansen, J. A.; Ajala, A. O. Benchmarking the completely renormalised equation-of-motion coupled-cluster approaches for vertical excitation energies. Mol. Phys. 2015, 113, $3085-3127$

(29) Huntington, L. M. J.; Demel, O.; Nooijen, M. Benchmark Applications of Variations of Multireference Equation of Motion Coupled-Cluster Theory. J. Chem. Theory Comput. 2016, 12, 114132.

(30) Rishi, V.; Perera, A.; Nooijen, M.; Bartlett, R. J. Excited states from modified coupled cluster methods: Are they any better than EOM CCSD? J. Chem. Phys. 2017, 146, 144104.

(31) Kozma, B.; Tajti, A.; Demoulin, B.; Izsák, R.; Nooijen, M.; Szalay, P. G. A New Benchmark Set for Excitation Energy of Charge Transfer States: Systematic Investigation of Coupled Cluster Type Methods. J. Chem. Theory Comput. 2020, 16, 4213-4225.

(32) Kozma, B.; Berraud-Pache, R.; Tajti, A.; Szalay, P. G. Potential energy surfaces of charge transfer states. Mol. Phys. 2020, 118, No. e1776903.

(33) Koch, H.; Jørgensen, P. Coupled cluster response functions. J. Chem. Phys. 1990, 93, 3333.

(34) Nooijen, M.; Bartlett, R. J. Similarity transformed equation-ofmotion coupled-cluster theory: Details, examples, and comparisons. The Journal of Chemical Physics 1997, 107, 6812-6830.

(35) Sekino, H.; Bartlett, R. J. A Linear Response, Coupled-Cluster Theory for Excitation-Energy. Int. J. Quantum Chem. 1984, 255-265.

(36) Stanton, J. F.; Bartlett, R. J. The Equation of Motion CoupledCluster Method. A Systematic Biorthogonal Approach to Molecular Excitation Energies, Transition Probabilities, and Excited-State Properties. J. Chem. Phys. 1993, 98, 7029-7039.

(37) Koch, H.; Jensen, H. J. A.; Jørgensen, P.; Helgaker, T. Excitation energies from the coupled cluster singles and doubles linear response function (CCSDLR). Applications to $\mathrm{Be}, \mathrm{CH}+, \mathrm{CO}$, and H2O. J. Chem. Phys. 1990, 93, 3345-3350.

(38) Christiansen, O.; Koch, H.; Jørgensen, P. Response Functions in the CC3 Iterative Triple Excitation Model. J. Chem. Phys. 1995, 103, 7429-7441.

(39) Grimme, S. Improved second-order Møller-Plesset perturbation theory by separate scaling of parallel- and antiparallel-spin pair correlation energies. J. Chem. Phys. 2003, 118, 9095-9102.

(40) Grimme, S.; Goerigk, L.; Fink, R. F. Spin-component-scaled electron correlation methods. Wiley Interdiscip. Rev.: Comput. Mol. Sci. 2012, 2, 886-906.

(41) Hellweg, A.; Grün, S. A.; Hättig, C. Benchmarking the performance of spin-component scaled $\mathrm{CC} 2$ in ground and electronically excited states. Phys. Chem. Chem. Phys. 2008, 10, 4119-4127.

(42) Winter, N. O. C.; Hättig, C. Scaled opposite-spin CC2 for ground and excited states with fourth order scaling computational costs. J. Chem. Phys. 2011, 134, 184101.

(43) Szabados, A. Theoretical interpretation of Grimme's spincomponent-scaled second order Møller-Plesset theory. J. Chem. Phys. 2006, 125, 214105.

(44) Winter, N. O. C.; Hättig, C. Quartic scaling analytical gradients of scaled opposite-spin CC2. Chem. Phys. 2012, 401, 217.

(45) Krauter, C. M.; Pernpointner, M.; Dreuw, A. Application of the scaled-opposite-spin approximation to algebraic diagrammatic construction schemes of second order. J. Chem. Phys. 2013, 138, No. 044107.

(46) Mester, D.; Nagy, P. R.; Kállay, M. Reduced-cost second-order algebraic-diagrammatic construction method for excitation energies and transition moments. J. Chem. Phys. 2018, 148, No. 094111.

(47) Mester, D.; Kállay, M. Combined Density Functional and Algebraic-Diagrammatic Construction Approach for Accurate Excitation Energies and Transition Moments. J. Chem. Theory Comput. 2019, 15, 4440-4453. 
(48) Balasubramani, S. G.; et al. TURBOMOLE: Modular program suite for ab initio quantum-chemical and condensed-matter simulations. J. Chem. Phys. 2020, 152, 184107.

(49) Shao, Y.; et al. Advances in molecular quantum chemistry contained in the Q-Chem 4 program package. Mol. Phys. 2015, 113, 184-215.

(50) Aidas, K.; et al. The Dalton quantum chemistry program system. WIREs Comput. Mol. Sci. 2013, 4, 269-284.

(51) Kállay, M.; et al. The MRCC program system: Accurate quantum chemistry from water to proteins. J. Chem. Phys. 2020, 152, No. 074107.

(52) Hellweg, A.; Grün, S. A.; Hättig, C. Benchmarking the performance of spincomponent scaled CC2 in ground and electronically excited states. Phys. Chem. Chem. Phys. 2008, 10, 4119-4127.

(53) Winter, N. O. C.; Graf, N. K.; Leutwyler, S.; Hättig, C. Benchmarks for $0-0$ transitions of aromatic organic molecules: DFT/ B3LYP, ADC(2), CC2, SOS-CC2 and SCS-CC2 compared to highresolution gas-phase data. Phys. Chem. Chem. Phys. 2013, 15, 66236630.

(54) Sauer, S. P. A.; Pitzner-Frydendahl, H. F.; Buse, M.; Jensen, H. J. A.; Thiel, W. Performance of SOPPA-based methods in the calculation of vertical excitation energies and oscillator strengths. Mol. Phys. 2015, 113, 2026-2045.

(55) Tajti, A.; Szalay, P. G. Accuracy of Spin-Component-Scaled CC2 Excitation Energies and Potential Energy Surfaces. J. Chem. Theory Comput. 2019, 15, 5523-5531.

(56) Tajti, A.; Tulipán, L.; Szalay, P. G. Accuracy of SpinComponent Scaled ADC(2) Excitation Energies and Potential Energy Surfaces. J. Chem. Theory Comput. 2020, 16, 468-474.

(57) Watts, J. D.; Bartlett, R. J. Iterative and non-iterative triple excitation corrections in coupled-cluster methods for excited electronic states: The EOM-CCSDT-3 and $\operatorname{EOMCCSD}(\tilde{\mathrm{T}})$ methods. Chem. Phys. Lett. 1996, 258, 581-588.

(58) Dunning, T. H., Jr. Gaussian basis sets for use in correlated molecular calculations. I. The atoms boron through neon and hydrogen. J. Chem. Phys. 1989, 90, 1007-1023.

(59) TURBOMOLE V7.3 2018, a development of University of Karlsruhe and Forschungszentrum Karlsruhe GmbH, 1989-2007, TURBOMOLE GmbH, since 2007; available from http://www. turbomole.com.

(60) Plasser, F.; Lischka, H. Analysis of Excitonic and Charge Transfer Interactions from Quantum Chemical Calculations. J. Chem. Theory Comput. 2012, 8, 2777-2789.

(61) Plasser, F.; Wormit, M.; Dreuw, A. New tools for the systematic analysis and visualization of electronic excitations I. Formalism. J. Chem. Phys. 2014, 141, No. 024106.

(62) Plasser, F.; Thomitzni, B.; Bäppler, S. A.; Wenzel, J.; Rehn, D. R.; Wormit, M.; Dreuw, A. Statistical analysis of electronic excitation processes: Spatial location, compactness, charge transfer, and electron-hole correlation. J. Comput. Chem. 2015, 36, 1609-1620.

(63) Plasser, F. TheoDORE: A toolbox for a detailed and automated analysis of electronic excited state computations. J. Chem. Phys. 2020, 152, No. 084108.

(64) Plasser, F.; Wormit, M.; Mewes, S. A.; Thomitzni, B.; Dreuw, A. LIBWFA: Wavefunction analysis tool library for quantum chemical applications; available from https://github.com/libwfa/libwfa.

(65) Stanton, J. F.; Gauss, J. A simple scheme for the direct calculation of ionization potentials with coupled-cluster theory that exploits established excitation energy methods. J. Chem. Phys. 1999, 111, 8785-8788.

(66) Pershin, A.; Szalay, P. G. Improving the Accuracy of the Charge Transfer Integrals Obtained by Coupled Cluster Theory, MBPT (2), and TDDFT. J. Chem. Theory Comput. 2015, 11, 5705-5711. 